\title{
ON A FRACTIONAL STOCHASTIC PATH INTEGRAL APPROACH IN MODELLING INTERNEURONAL CONNECTIVITY
}

\author{
CHRISTOPHER C. BERNIDO AND M. VICTORIA CARPIO-BERNIDO \\ Research Center for Theoretical Physics, Central Visayan Institute Foundation, Jagna, \\ Bohol 6308, Philippines \\ E-mail: cbernido@mozcom.com
}

\begin{abstract}
A fractional stochastic path integral approach is presented as a natural framework for treating the random distribution of possible communication chains in the synaptic transmission of signals between initiator and distant target receptor neurons. Fractional Brownian motion parametrization is invoked to account for strong correlations between segments of a neuronal communication chain. We then obtain the probability density function (pdf) for the location of the target receptor neuron in terms of the Hurst index that classifies the dynamics into short-memory or long-memory domains. This pdf obtained by the path integral approach is a fundamental solution of the corresponding Fokker-Planck equation.
\end{abstract}

\section{Introduction}

In this report, we present a fractional stochastic path integral approach to derive the conditional probability density function (pdf) for the propagation of neuronal signals through interconnected chains of neurons. A number of considerations make this approach interesting as a source of insight in the investigation of current core problems of neuroscience, i.e., connectivity, memory, correlations and synchrony. In particular, we note some salient properties of biological systems: (i) Populations of neurons are typically extremely dense, with a single neuron possibly linked to around 10,000 other neurons through a complex outlay of dendritic arborizations and synaptic branching., ${ }^{1,2}$ (ii) An enormous number of possible link configurations for the synaptic transmission of signals between initiator and distant target receptor neurons, with an individual neuron typically also receiving signals from other neurons that are not part of the chain directly linking initiator and final receiver neurons. Indeed, a multitude of input signals (which could be over 30,000 synaptic inputs for a single pyramidal cell) are integrated by a neuron for each output signal it sends out to adjacent neurons in a chain. (iii) Interneuronal connectivity is determined by synaptic activity that is highly variable and dynamic. At the cellular level, various experiments, indicate that memory is encoded by changes in the synaptic strength or connectivity between communicating neuronal cells in the brain. ${ }^{3-6}$ These considerations lead us to an analytical framework whose elements 
could be matched vis-á-vis biological features. First, we use the Feynman path integral approach since it precisely works for systems with large numbers of degrees of freedom, having been conceptually designed to work as a 'summation over all possible histories' of a physical system. Second, a stochastic description appears particularly suitable since lines of communication within networks of neurons are not deterministic in the sense of well-defined and predictable trajectories for signal transmission. We thus turn to a rigorous formulation of the sum over all paths as a generalized stochastic functional integral over the infinite-dimensional white noise space of distributions introduced by Streit and Hida. ${ }^{7,8} \mathrm{~A}$ distinct characteristic of the path integral approach would be its being global in nature, in contrast to other network and graph-theoretical approaches that work bottom-up starting with the simplest point-to-point connections, and inductively going up to more connections and branches. Clearly, the latter would be bound to encounter increasing demand for computational time and power to comprehensively handle increasingly complex neuronal architecture. Moreover, the pdf obtained with the path integral approach is a propagator or Green function which is also the fundamental solution of the corresponding Fokker-Planck equation. On top of these, the global nature of the integral makes it convenient for handling boundary conditions and effects of topological constraints. ${ }^{9}$ Third, the propagation of signals or points in the interneuronal flow of information can be endowed with memory, with each point along a signal transmission trajectory depending on an earlier neuronal configuration. Thus, we parametrize fluctuations in the path integral in terms of fractional Brownian motion $(\mathrm{fBm})$ characterized by the Hurst index $H$. An advantage of $\mathrm{fBm}$ is its being essentially non-Markovian, having long-range spatial and temporal correlations. ${ }^{10}$ One can endow neuronal connections with memory, and classify paths into those with long-memory properties $(1 / 2<H<1)$, and those involving short-memory processes $(0<H<1 / 2)$. For $H=1 / 2$, we recover ordinary Brownian motion. The mathematical foundations of $\mathrm{BBm}$ have been developed to a high level which allows easier application to physical and biological systems. ${ }^{11-13}$

Our treatment here differs from earlier works with stochastic analysis or path integrals, e.g., (1) random walk models for the activity of single neurons, ${ }^{14}$ (2) stochastic jump processes for the dynamics of neuronal membrane depolarization potentials, ${ }^{15}$ (3) path integral framework for evaluating the partition function for whole neural network dynamics, ${ }^{16}$ (4) path integral evaluation of the potential at different points along dendritic trees, ${ }^{17}$ (5) an algorithm for evolution of short-term memory in the statistical mechanics of neocortical interactions, ${ }^{18}$ (6) path integral for the propagation of cerebellar signals, ${ }^{19}$ and (7) spin coherent state path integrals for the neural network master equation describing neural activity. ${ }^{20}$ Finally, the way we implement fBm parametrization differs from earlier works on the fractal features of neuronal structures and behavior as reviewed in Ref. 21.

In the next section, we set up the fractional path integral for the propagation of signals between neurons, followed by its analytical evaluation for the pdf. In section 4, we discuss information on neuronal connectivity and correlations that can 
be derived from the pdf. We can then look at how these properties could change as one studies connectivity in a local cluster, modelling, for example, one cubic millimeter of brain tissue. We could also draw insights as to how to extrapolate to macroscales where neuronal connectivity can be in different anatomical areas. ${ }^{22}$ This is important since, as pointed out in Ref. 23, a transmission line linking single neurons may be unreliable. The last section then describes how, using the characteristic self-similarity properties of $\mathrm{fBm}$, we can scale up to treat communication between neuronal populations. We end with some concluding remarks.

\section{Fractional Stochastic Path Parametrization for Connections with Memory}

In view of the high density of neuronal connections, a large number of possible neuronal communication chains may conduct signals starting from an initiator neuron at $\mathbf{r}_{0}$, through intermediate neurons with their corresponding axons, synapses and dendrites, and finally received by a target receptor neuron at $\mathbf{r}$. One could then visualize a random distribution of signal transmission trajectories or diffusion paths starting at $\mathbf{r}_{0}$ and ending at $\mathbf{r}$. Figure 1 shows two possible communication trajectories. Note that, for biological systems, neurons which are part of the communication chain may also receive signals from other neurons that are not part of the chain connecting $\mathbf{r}_{0}$ and $\mathbf{r}$. A multitude of input signals (which could be over 30,000 synaptic inputs for a single pyramidal cell) are integrated by a neuron for the resulting output signals it sends to adjacent neurons in a chain. Here, however, we are considering only the transmission of actual output signals. Any variations in the information would be carried by the $\mathrm{fBm}$ parametrization. Thus, we have for the diffusion paths,

$$
\mathbf{r}(s)=\mathbf{r}_{0}+\mathbf{B}^{H}(s),
$$

where we note that, the parameter $s, 0 \leq s \leq L$, is a segmental path length which increases at each segment or step of the fBm and $L$ is a total path length for the connections between $\mathbf{r}(0)$ and $\mathbf{r}(L), \mathbf{r}=(x, y, z)$. This parametrization, in lieu of time $t$, is allowed by defining short path intervals $\Delta s \approx v t$, where $v$ is an average velocity of Brownian motion. This is in analogy to the path integral parametrization of polymer chains in terms of monomer lengths by Edwards. ${ }^{24}$ The $\mathbf{B}^{H}=\left(B_{x}^{H}, B_{y}^{H}, B_{z}^{H}\right)$ with components $B_{i}^{H}(i=x, y, z)$, is a fBm in the RiemannLiouville fractional integral representation defined by, ${ }^{13}$

$$
B_{i}^{H}(L)=\frac{1}{\Gamma\left(H+\frac{1}{2}\right)} \int_{0}^{L}(L-s)^{H-\frac{1}{2}} d B_{i}(s) .
$$

Note that the derivative of the Brownian motion, $\omega_{i}=d B_{i} / d s$, which is white noise, may also be used to write $d B_{i}=\omega_{i} d s$ in Eq. (2). The Hurst index $H$ describes fBm with long-memory property (enhanced diffusion) for values, $1 / 2<H<1$, and 
short-memory processes (suppressed diffusion) for, $0<H<1 / 2$. The case $H=1 / 2$ describes the Wiener process or ordinary Brownian motion.

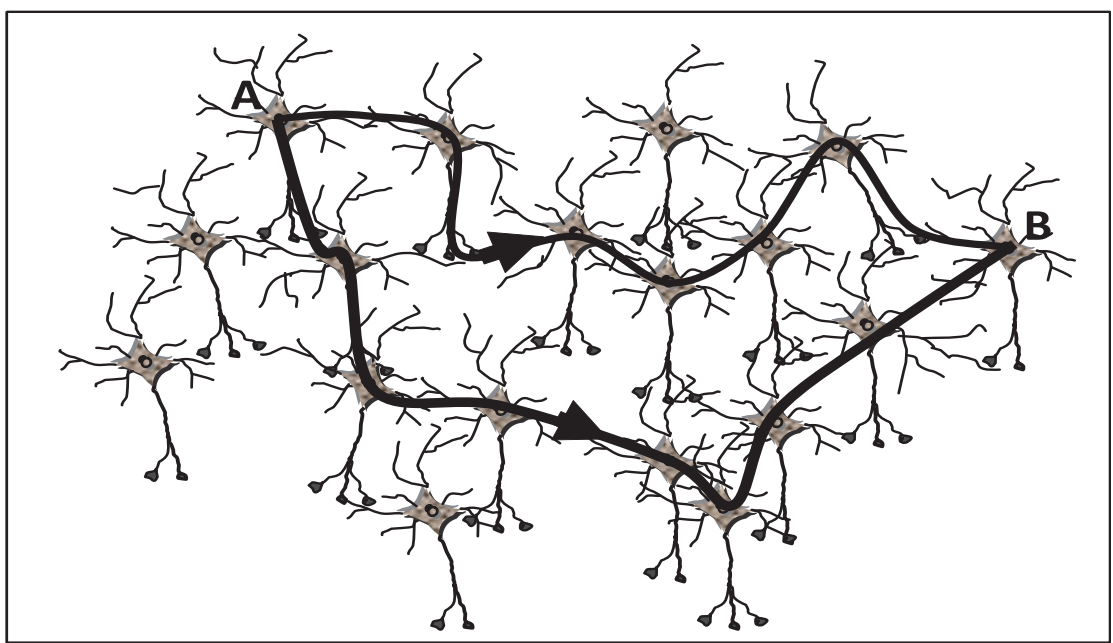

Fig. 1. There are numerous possible paths linking communicating neurons, for example, the top and bottom paths linking neurons $\mathrm{A}$ and $\mathrm{B}$.

\section{Evaluation of the Probability Density Function}

We now consider the sum-over-all possible connections or configurations of paths $\mathbf{r}(s)(0 \leq s \leq L)$ that start with an initiator neuron located at $\mathbf{r}(0)=\mathbf{r}_{0}$ and end at the target receptor neuron located at $\mathbf{r}(\mathbf{L})=\mathbf{r}_{L}$. Specifically, we allow all possible paths described by Eq. (1) subject to the constraint of terminating at $\mathbf{r}_{L}$. In other words, regardless of their configuration, contributing paths are only those that satisfy the constraint, $\delta\left(\mathbf{r}(s)-\mathbf{r}_{L}\right)$. What then is the distribution probability for all these connecting paths which satisfy the constraint? The conditional probability density function $P\left(\mathbf{r}_{L}, \mathbf{r}_{0} ; L\right)$, gives the probability that the position $\mathbf{r}(s)$ is $\mathbf{r}_{L}$ at $s=L$, given the initial condition that its value is $\mathbf{r}_{0}$ at $s=0$. Its value can be obtained by evaluating the expectation value of the delta function constraint, i.e.,

$$
P\left(\mathbf{r}_{L}, \mathbf{r}_{0} ; L\right)=E\left(\delta\left(\mathbf{r}(s)-\mathbf{r}_{L}\right)\right)=\int \delta\left(\mathbf{r}(s)-\mathbf{r}_{L}\right) d \mu
$$

where $d \mu$ is a Gaussian white noise measure defined by the characteristic functional, ${ }^{8}$

$$
C(\xi)=\int_{E *} \exp [i<x, \xi>] d \mu(x)=\exp \left[-\frac{1}{2}\|\xi\|^{2}\right], \quad \xi \in E .
$$

Here, $\|\cdot\|$ denotes the usual norm on the Hilbert space of square integrable functions and $E$ is the space of test functions in the Gelfand triple, $E \subset L^{2} \subset E^{*}$. The 
probability density is then expressed as a weighted average over fBm paths with end-point pinning. Considering the $x$ degree of freedom, for simplicity, we have,

$$
P\left(x_{L}, x_{0}\right)=\int \delta\left(x_{0}+B_{x}^{H}-x_{L}\right) d \mu_{x}
$$

Writing the delta function in terms of its Fourier representation we have,

$$
\begin{aligned}
P\left(x_{L}, x_{0}\right)= & \int\left(\frac{1}{2 \pi} \int_{-\infty}^{+\infty} \exp \left\{i k\left[\left(x_{0}-x_{L}+B_{x}^{H}\right)\right]\right\} d k\right) d \mu_{x} \\
= & \frac{1}{2 \pi} \int_{-\infty}^{+\infty} d k \exp \left\{i k\left[\left(x_{0}-x_{L}\right)\right]\right\} \\
& \times \int \exp \left\{i k \int_{0}^{L} \frac{(L-s)^{H-\frac{1}{2}} \omega_{x} d s}{\Gamma\left(H+\frac{1}{2}\right)}\right\} d \mu_{x} .
\end{aligned}
$$

The integration over $\omega_{x}$ appears as a Fourier transform of $d \mu_{x}$, i.e.,

$$
\begin{aligned}
\int \exp \left\{i k \int_{0}^{L} \frac{(L-s)^{H-\frac{1}{2}} \omega_{x} d s}{\Gamma\left(H+\frac{1}{2}\right)}\right\} d \mu_{x} & =\int \exp \left\{i \int_{0}^{L} f(s) \omega_{x}(s) d s\right\} d \mu_{x} \\
& =\exp \left\{-\frac{1}{2} \int_{0}^{L} f(s)^{2} d s\right\},
\end{aligned}
$$

where the right-hand-side is just the characteristic functional ${ }^{8}$ and,

$$
f(s)=\frac{k(L-s)^{H-\frac{1}{2}}}{\Gamma\left(H+\frac{1}{2}\right)} .
$$

Integrating $\int f(s)^{2} d s$ in Eq. (6) and using this in Eq. (5) we have,

$$
P\left(x_{L}, x_{0}\right)=\frac{1}{2 \pi} \int_{-\infty}^{+\infty} \exp \left\{i k\left(x_{0}-x_{L}\right)\right\} \exp \left\{-\frac{k^{2} L^{2 H}}{4 H \Gamma^{2}\left(H+\frac{1}{2}\right)}\right\} d k
$$

The remaining Gaussian integral can be evaluated to yield,

$$
P\left(x_{L}, x_{0}\right)=\sqrt{\frac{H \Gamma^{2}\left(H+\frac{1}{2}\right)}{\pi L^{2 H}}} \exp \left\{-\frac{H \Gamma^{2}\left(H+\frac{1}{2}\right)\left(x_{L}-x_{0}\right)^{2}}{L^{2 H}}\right\} .
$$


The $P\left(y_{L}, y_{0}\right)$ and $P\left(z_{L}, z_{0}\right)$ for the $y$ and $z$ degrees of freedom can be obtained with a similar calculation as that for $P\left(x_{L}, x_{0}\right)$. In the following section, it would be sufficient to consider the properties of $P\left(x_{L}, x_{0}\right)$.

Note that the probability distribution function $P\left(x_{L}, x_{0}\right)$, with $0 \leq s \leq L$, obeys an effective Fokker-Planck equation of the form, ${ }^{25}$

$$
\frac{\partial}{\partial s} P(x, s)=\frac{s^{2 H-1}}{2 \Gamma^{2}\left(H+\frac{1}{2}\right)} \frac{\partial^{2}}{\partial x^{2}} P(x, s) .
$$

When the Hurst index is $H=1 / 2$, Eq. (10) reduces to the usual diffusion equation for Brownian motion.

\section{Discussion}

\subsection{Distance and Connectivity between Neurons}

What information can be extracted from the probability distribution function $P\left(x_{L}, x_{0}\right)$ ? If we designate, $\mathbf{x}=x_{L}-x_{0}$, as the physical distance along the $x$ axis between the neurons at $\mathbf{r}_{0}$ and $\mathbf{r}_{L}$, we can graph the probability distribution function, Eq. (9), as a function of distance x. In particular, we can do this for three different values of the Hurst index (see, Fig. 2). In the figure, the solid line is for $H=0.5$ (ordinary Brownian motion), while the dashed line, $H=0.3$, represents connections modelled by a suppressed diffusion. The dotted line, $H=0.8$, describes paths for the case of enhanced diffusion. All three graphs are for the same total path length $L$.

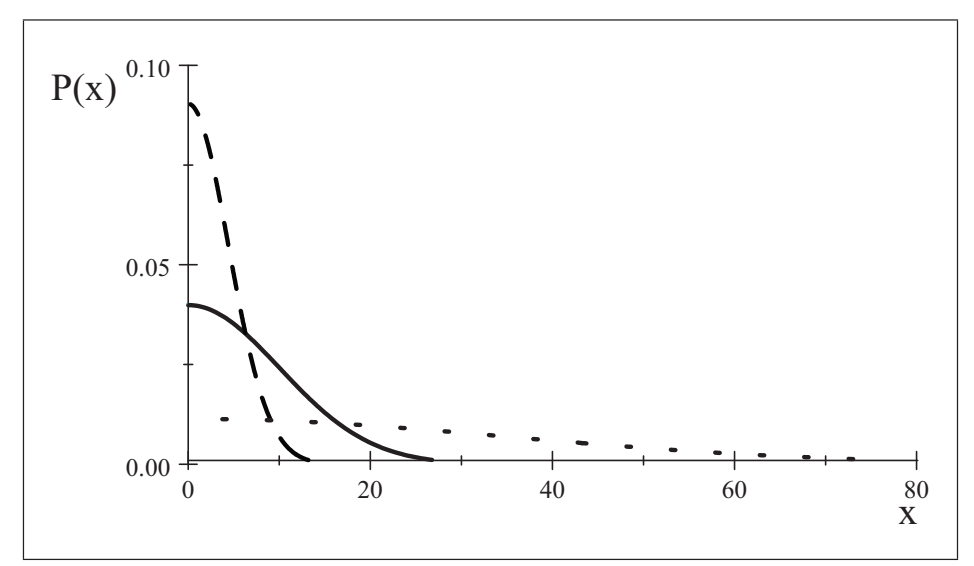

Fig. 2. Solid line: $H=0.5$ (ordinary Brownian motion); Dash: $H=0.3$; Dots: $H=0.8$. Two neurons are more likely to be far apart when $0.5<H<1$.

As exemplified by the curves in Fig. 2, if two neurons that are near each other (i.e., small values of $\mathbf{x}=x_{L}-x_{0}$ ) communicate through neuronal chains of path length $L$, the connections involved would more likely correspond to the sub-diffusion 
type $(0<H<0.5)$. On the other hand, two communicating neurons far from each other (i.e., large $\mathbf{x}$ ), such as those located at different regions of the brain would likely have connection pattterns corresponding to an enhanced diffusion process $(0.5<H<1)$.

Equation (9) therefore indicates that connectivity could be scale-dependent where nearby neurons (described by $0<H<0.5$ ) and neurons significantly separated (e.g., for $0.5<H<1$ ) have different connectivity patterns characterized by the Hurst index $H$. This reminds us of a recent experiment with multiple-tetrode recordings from primary visual cortex of macaque monkeys which showed a scaledependent structure of cortical interactions. ${ }^{26}$ The results showing how connectivity is organized in the cortex at multiple scales were based on recordings where a tetrode isolates several neurons within a radius of $\sim 150 \mu \mathrm{m}$, monitoring small groups of 3 to 6 neurons, as well as tetrodes separated by distances ranging from $600 \mu \mathrm{m}$ to several $\mathrm{mm}$. The investigators pointed out a curious result: unlike neurons separated by large distances, local clusters of neurons exhibited flexible correlations that are rapidly reorganized by visual input. In our framework, local clusters would correspond to short memory processes where $0<H<0.5$.

\subsection{Correlation and Synchrony between Neuronal Activity}

The correlation of spiking neurons, both at the temporal and spatial scales, has been the subject of many experimental investigations. The spatial properties and distance between correlated neurons, however, appears to be an important factor in understanding the mechanism for correlation. Recent technological advances in using multielectrode arrays ${ }^{27}$ and calcium imaging techniques ${ }^{28}$ have allowed measurement of correlation between activities of neuron pairs in vivo. With multiple tetrode recordings, ${ }^{26}$ for instance, firing patterns can be compared among local clusters of neurons $(<300 \mu \mathrm{m}$ apart) with those of neurons separated by larger distances $(600-2,500 \mu \mathrm{m})$. Results show that firing patterns at larger distances are predicted by pairwise interactions, while patterns within local clusters show evidence of high-order correlations. Correlations are taken to manifest primarily in synchronized firing of neurons, that is when two or more spiking neurons fire jointly. ${ }^{23}$ It is thus of interest to study both short-range and long-range communication between neurons separated by some distance or by layers of intermediate neurons. Furthermore, synchrony was found evident between nearby neurons separated by $3 \mathrm{~mm}$ or less, while correlated variability decreased slowly with distance but is still significant between neurons separated by 10 millimeters.

In the present formalism where we idealized the connections as $\mathrm{BBm}$ paths, the correlation between two neurons at points along the chain can be calculated. In particular, the expectation value or correlation is of the form, ${ }^{13}$

$$
E\left(x\left(s_{1}\right) x\left(s_{2}\right)\right) \simeq \frac{1}{2}\left(\left|s_{1}\right|^{2 H}+\left|s_{2}\right|^{2 H}-\left|s_{1}-s_{2}\right|^{2 H}\right),
$$


where $s_{1}$ and $s_{2}$ are distance parameters specifying the locations of two correlated neurons along the contour path length (see, Fig. 3). Equation (11) tells us that correlation largely depends on distances of neurons along the path length. In particular, for two neighboring neurons where $s_{1} \approx s_{2}$, the negative term, $\left|s_{1}-s_{2}\right|^{2 H}$ is negligible resulting in a relatively higher correlation. On the other hand, if two neurons have a large separation along the path length, i.e. $\left|s_{1}-s_{2}\right|^{2 H}$ becomes large in Eq. (11), the correlation between neurons could dramatically decrease. This could give insight on the interpretation of results of experiments showing that correlated neuronal activities depend on the scale. For instance, sampling of cortical activities at different scales showed firing patterns for local clusters of neurons $(<300 \mu \mathrm{m}$ apart) with high-order correlations, which differed from neurons separated by larger distances $(600-2,500 \mu \mathrm{m})$ which can be predicted by pairwise interactions. ${ }^{26}$

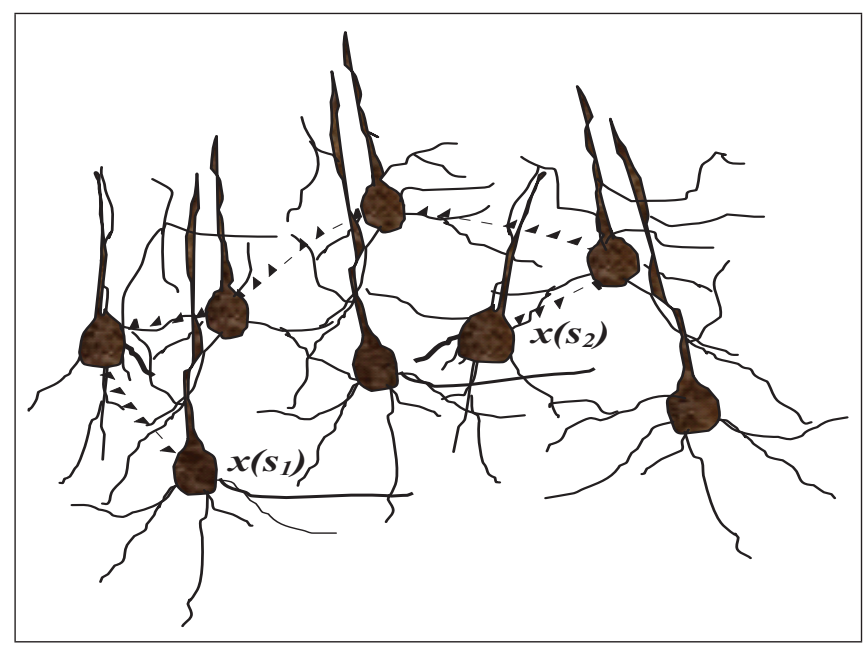

Fig. 3. Two neurons at $x\left(s_{1}\right)$ and $x\left(s_{2}\right)$, which could be physically near each other, may communicate using a much longer path length $L$ (dashed line).

Equation (11) also depends on the value of the Hurst index $H$ which indicates whether the connection stucture is of the suppressed or enhanced type of diffusion. In particular, as observed in the previous section, two communicating neurons which are physically near each other (small values of $\mathbf{x}$, but may have large contour separation $\left|s_{1}-s_{2}\right|$ ), would have connections that are more likely to be sub-diffusive.

The mean squared distance between two points of the neuronal chain can likewise be computed and is given by,

$$
E\left(\left[x\left(s_{1}\right)-x\left(s_{2}\right)\right]^{2}\right)=\left|s_{1}-s_{2}\right|^{2 H} .
$$

Again, we have to distinguish between the actual physical distance between two neurons given by, $x\left(s_{1}\right)-x\left(s_{2}\right)$ along the $x$-axis, and their separation along the 
contour path given by the length, $\left|s_{1}-s_{2}\right|$. Two neighboring neurons $\left(x\left(s_{1}\right) \approx\right.$ $\left.x\left(s_{2}\right)\right)$ may have a large separation, $\left|s_{1}-s_{2}\right|$, along the path length (see, Fig. 3 ).

Equation (12), can further be generalized for higher moments, $n \geq 1$, to yield the result, ${ }^{13}$

$$
E\left(\left[x\left(s_{1}\right)-x\left(s_{2}\right)\right]^{n}\right)=\frac{2^{\frac{n}{2}}}{\sqrt{\pi}} \Gamma\left(\frac{n+1}{2}\right)\left|s_{1}-s_{2}\right|^{n H} .
$$

The expression, Eq. (13), tells us that even if two neurons are physically very close to each other, i.e., $x\left(s_{1}\right) \approx x\left(s_{2}\right)$, the expectation value of $\left[x\left(s_{1}\right)-x\left(s_{2}\right)\right]^{n}$ could be large if their separation along the contour length, $\left|s_{1}-s_{2}\right|$, is large. Equation (13) may be checked with empirical investigations of paired neurons and higher-order correlations.

\subsection{Scaling Property for Neuronal Clusters}

One of the challenges facing theoretical and experimental investigations in neuroscience is the tracking of activities between populations of neurons. Here one essentially goes from studying small groups of connected neurons, to bigger clusters or assemblies of neurons, all the way up to connected large neuron populations. The connectivity to be investigated this time involves a neuron population receiving inputs from a previous neuron population as it projects to a subsequent neuron population (see, Fig. 4).

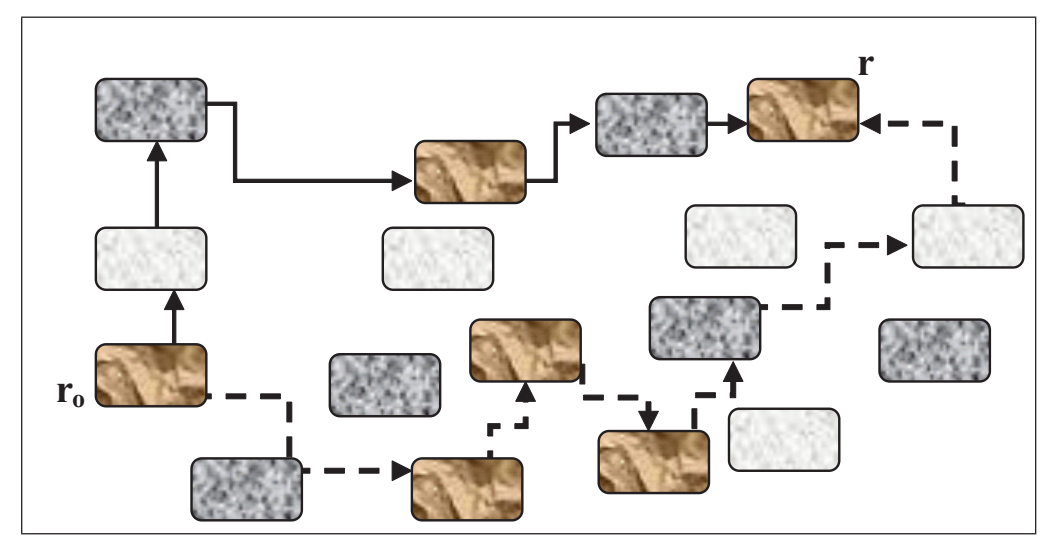

Fig. 4. Each rectangle represents a neuron population.

This is closer to biologically realistic models with relationships between local activities of neurons linked by synapses to large-scale global connectivity involving networks of brain regions connected by anatomical tracts. The inherent self-similarity property of fBm allows the present formulation to offer a way of tracking small-scale neuronal activities to large-scale activities between neuron populations. One could 
implement a description of neuronal connectivity by introducing a scale factor $b>0$ such that the path length $s$ and distance along $x$ transform as,

$$
s \rightarrow b s \quad ; \quad x \rightarrow b^{H} x .
$$

It is easy to check that under the transformations given by Eq. (14), the probability distribution function Eq. (9) transforms accordingly as,

$$
P\left(b^{H} x_{s}, b^{H} x_{0} ; b s\right)=b^{-H} P\left(x_{s}, x_{0} ; s\right) .
$$

Equation (15) states that the probability distribution function for connectivities on the large scale described by $P\left(b^{H} x_{s}, b^{H} x_{0} ; b s\right)$ has the same property and behavior as the small-scale probability distribution function $P\left(x_{s}, x_{0} ; s\right)$ except for a factor of $b^{-H}$. Scale transformations may describe connectivity between neuron populations. Although anatomically segregated regions of the brain are highly specialized in their functions, a self-similarity property may, perhaps, help characterize the structure and function of the brain.

\section{Conclusion}

In this report, we presented a stochastic sum-over-all-paths approach and modelled neuronal connections with memory by using the fractional Brownian motion (fBm) representation. Within this framework we obtained the probability distribution that yields various expressions for pairwise correlation of neurons relevant to some recent experimental results. Furthermore, we noted that, in view of its self-similarity property, the sum-over-all histories of fBm paths may also be a useful tool in studies involving interactions between populations of neurons. This is a step towards understanding collective neuronal behavior important for questions that arise in problems of learning and memory.

\section{Acknowledgments}

The authors are grateful to the Alexander von Humboldt Foundation for supporting our visit at the Bernstein Center Freiburg, University of Freiburg, where we had stimulating discussions with the Computational Neuroscience group led by Prof. Dr. Ad Aertsen. CCB also acknowledges the support of the National Academy of Science and Technology (Philippines).

\section{References}

1. T. Binzegger, J. Neurosci. 24, 8441 (2004).

2. V. Braitenberg and A. Schüz, Statistics and Geometry of Neuronal Connectivity (Springer-Verlag, Berlin, 1998).

3. M. Sanhueza et al, Jour. Neurosci. 31, 9170 (2011). 
4. J. A. Kleim, et al., Proc. Natl. Acad. Sci. 99, 13228 (2002).

5. J. A. Deutsch, Science 174, 788 (1971).

6. S. J. Martin, P. D. Grimwood, R. G. Morris, Annu. Rev. Neurosci. 23, 649 (2000).

7. L. Streit and T. Hida, Stoch. Proc. Appl. 16, 55 (1983).

8. T. Hida, H. H. Kuo, J. Potthoff, L. Streit, White Noise. An Infinite Dimensional Calculus (Kluwer, Dordrecht, 1993).

9. C. C. Bernido and M. V. Carpio-Bernido, J. Math. Phys. 43, 1728 (2002).

10. H. Qian, In Processes with Long-range Correlations: Theory and Applications, Lecture Notes in Physics, Vol. 621, Eds., G. Rangarajan and M. Z. Ding, p. 22 (Springer, Berlin, 2003).

11. B. Mandelbrot, and J.W. van Ness, SIAM Review 10 (4), 422 (1968).

12. K. S. Miller and B. Ross, An Introduction to the Fractional Calculus and Fractional Differential Equations (John Wiley \& Sons, New Jersey, 1993).

13. Y. Mishura, Stochastic Calculus for Fractional Brownian Motion and Related Processes, (Springer-Verlag, Berlin, 2008).

14. G. L. Gerstein and B. Mandelbrot, Biophysical Jour. 4, 41 (1964).

15. P. Blanchard, Ph. Combe, H. Nencka, and R. Rodriguez, J. Math. Biol. 31, 189 (1993).

16. J. Balakrishnan, arXiv:cond-mat/0308503v1 [cond-mat.stat-mech] (2003).

17. L. F. Abott, E. Farhi and S. Gutman, Biol. Cyber. 66, 49 (1991).

18. A L. Ingber, Phys. Rev. E, V 49, N. 5, 4652 (1994).

19. R. E. Shaw, E. E. Kadar and M. T. Turvey, Behavioral and Brain Sciences 20, 265 (1997).

20. T. Ohira and J. D. Cowan, in Proc. World Congress on Neural Networks, San Diego, June 1994 (Sony Computer Science Lab. Inc. 1995).

21. G. Werner, Frontiers in Physiology, 1 (2010) doi: 10.3389/fphys.2010.00015.

22. N. Voges, A. Schüz, A. Aertsen, S. Rotter, Progress in Neurobiology 92, 277 (2010).

23. See, e.g., A. Kumar, S. Rotter, and A. Aertsen, Nature Rev.11, 615 (2010).

24. M. Doi and S. F. Edwards, The Theory of Polymer Dynamics (Oxford Univ. Press, New York, 1986).

25. I. Calvo and R. Sánchez, J. Phys. A: Math. Theor. 41, 282002 (2008).

26. I. E. Ohiorhenuan, F. Mechler, K. P. Purpura, A. M. Schmid, Q. Hu and J. D. Victor, Nature 466, 617 (2010).

27. M. A. Smith and A. Kohn, J. Neurosci. 28, 12591 (2008).

28. Y. H. Ch'ng and R. C. Reid, Front. Integr. Neurosci. 4, 1 ( 2010). 